\title{
Multi-Objective Optimal Control of the 6-DoF Aeroservoelastic Common Research Model with Aspect Ratio 13.5 Wing
}

\author{
Michael Drew* \\ Stinger Ghaffarian Technologies, Inc., Moffett Field, CA 94035 \\ Kelley E. Hashemi ${ }^{\dagger}$ \\ Universities Space Research Association, Moffett Field, CA 94035 \\ Nicholas Cramer \\ Stinger Ghaffarian Technologies, Inc., Moffett Field, CA 94035 \\ Nhan T. Nguyen ${ }^{\S}$ \\ NASA Ames Research Center, Moffett Field, CA 94035
}

\begin{abstract}
A new 6-DoF aeroservoelastic (ASE) Common Research Model (CRM) provided by The Boeing Company with aspect ratio 13.5 and 17 control surfaces per wing is utilized to demonstrate combined tracking and optimal multi-objective control. The multi-objective controller is derived on the closed loop tracking controller, and utilizes state and gust estimates provided by an extended state observer. Various methods of model reduction useful for control and estimation are presented. A computationally efficient MATLAB/Simulink simulation is presented which includes actuator dynamics, rate and deflection saturation limits, and gust disturbance inputs. The platform is used to demonstrate excellent 6-DoF tracking control performance coupled with the multi-objective controller, which is shown to effectively reduce structural mode movement, wing root bending moment, and drag. State and gust estimation is also shown to perform well, even when derived and/or implemented with significantly fewer states than the original full-sized model.
\end{abstract}

\section{Introduction}

S aircraft manufacturers seek ways to reduce weight and increase efficiency by using lighter materials, the Aresulting loss of structural rigidity may decrease the aerodynamic performance, leading to increased drag, reduced flutter margins, and undesired loading. In recent years, research has been investigating Performance Adaptive Aeroelastic Wing (PAAW) technology, which incorporates novel control surface designs and control algorithms to actively shape the wing in order to mitigate these issues. One proposed technology, known as Variable Camber Continuous Trailing Edge Flap (VCCTEF), uses several small multi-cambered control surfaces on each wing's trailing edge ${ }^{1}$ to shape the span-wise lift distribution of the wing. This is a concept developed under NASA's Advanced Air Transport Technology (AATT) project, and significant research has been conducted in this area, the bulk of which has used a longitudinal dynamic model of the Generic Transport Model (GTM). Ref. 2, for example, presents an aeroservoelastic (ASE) model of the GTM with VCCTEF, along with simulation results of a multi-objective optimal controller that provides drag reduction, maneuver load reduction, and flutter suppression.

In this paper, the authors present a new application of these control strategies applied to a full 6-Degreesof-Freedom (DoF) Common Research Model (CRM) with an aspect ratio of 13.5. This modified form of

\footnotetext{
* Research Engineer, Stinger Ghaffarian Technologies, Inc., NASA Ames Research Center, michael.c.drew@nasa.gov

${ }^{\dagger}$ NASA Post-Doctoral Fellow, Intelligent Systems Division, kelley.e.hashemi@nasa.gov

${ }^{\ddagger}$ Research Engineer, Stinger Ghaffarian Technologies, Inc., NASA Ames Research Center, nicholas.b.cramer@nasa.gov

$\S$ Research Scientist, Intelligent Systems Division, nhan.t.nguyen@nasa.gov, AIAA Associate Fellow
} 
the existing CRM (with $A R=9$ ) features additional trailing edge control surfaces with the design intent of suppressing the undesirable side effects that may accompany the use of a more efficient (and potentially softer) high aspect ratio wing design. A nominal controller is presented that tracks both heading and flight path angles. Then, a multi-objective optimal controller is developed that suppresses structural motion, provides gust and maneuver load alleviation, and reduces drag.

This paper begins in Section II with a brief overview and description of both the original CRM and the version used for this work. Section III describes the several versions of the linear CRM model provided by Boeing. Section IV presents the nominal tracking controller used for tracking heading and flight path angle. In Section V the multi-objective controller is derived. Model reduction as well as state and gust estimator designs are discussed in Section VI. Full multi-objective simulation results are presented in Section VII, with conclusions and future research directions detailed in Section VIII.

\section{Background}

For about a decade, NASA's Common Research Model (CRM) has been a useful tool for experimentally validating specific Computational Fluid Dynamics (CFD) predictions on many items including control surface effectiveness, trim drag, and stability derivatives. As a common model, it has also been useful for crossvalidating experimental results across numerous wind tunnels, helping to anchor measurements and correction methods between facilities. See Ref. 3 for example.

The original Common Research Model (CRM) was developed in 2008 as a joint effort between The Boeing Company, which led the aerodynamic design, and NASA, which was charged with fabricating and testing the design. The goal was to create a modern airframe geometry similar in form factor to a transonic wide-body commercial transport aircraft, but to be publicly available as a research tool. It was chosen to have a cruise Mach number of $M=0.85$, with a nominal lift condition of $C_{L}=0.50$ at a Reynolds number of $R e=40$ million per reference chord. The wing was specified to have an aspect ratio of 9.0 and was modeled in a deflected shape corresponding to a $1 \mathrm{~g}$ cruise flight condition.

With the motivation of studying the performance and control of higher aspect ratio aircraft, a modified version of the CRM was created by Boeing's Research \& Technology group with an aspect ratio of 13.5, herein called the CRM-13.5. Similar to the GTM VCCTEF concept, the CRM-13.5 features 17 trailing edge control surfaces on each wing, as shown in Fig. 1. Both a full scale mathematical model has been developed, along with a $10.8 \%$ geometric scale physical model for future wind tunnel testing at NASA Langley Research Center. Like the CRM-9, the CRM-13.5 used in this work is modeled in its deflected shape at the nominal $1 \mathrm{~g}$ flight condition. Recently, undeflected variants of both aspect ratio models (known as uCRM-9 and uCRM-13.5) have been created for specific aerostructural research. Further information on the development and technical specifications of the original CRM can be found in Ref. 4, and a description of the undeflected variants can be found in Ref. 5 .

\section{The CRM-13.5 Model}

The Boeing Company has provided linear state space models of the full scale CRM-13.5 at 60 trim conditions representing various altitudes, Mach numbers, and weights. Included at each trim point are three rigid body models (lateral, longitudinal, and combined), and a "blended model" that combines rigid body and aeroelastic dynamics. The blended model, which has 491 states, is considered to be the highest fidelity and includes 9 rigid body states, 27 structural modes, 6 aeroelastic lag states per mode, 6 lag states per the 36 control surfaces, and 8 states related to gust disturbance effects.

A separate second-order model is also provided representing the actuator dynamics of the 36 control surfaces (17 per wing + elevator and rudder). The actuator model is identical for all trim conditions, and has as its inputs the commanded deflection for each control surface. The outputs consist of "actual" control surface deflections, rates, and accelerations, which are used as inputs to the blended model. For this work, additional saturation constraints on the actuators are enforced; deflection is limited to a range of $\pm 25^{\circ}$, and deflection rate to $\pm 60^{\circ} \mathrm{s}^{-1}$. The actuator model also provides a channel for a one-dimensional vertical wind velocity gust disturbance signal. It produces low-pass filtered (having a $16 \mathrm{~Hz}$ cutoff frequency) vertical gust velocity and acceleration signals for inputs to the aircraft model. 266 outputs are available from the aircraft model, including $19 x, y, z$ displacement and acceleration outputs on each wing. Sensor dynamics and noise are not included. 


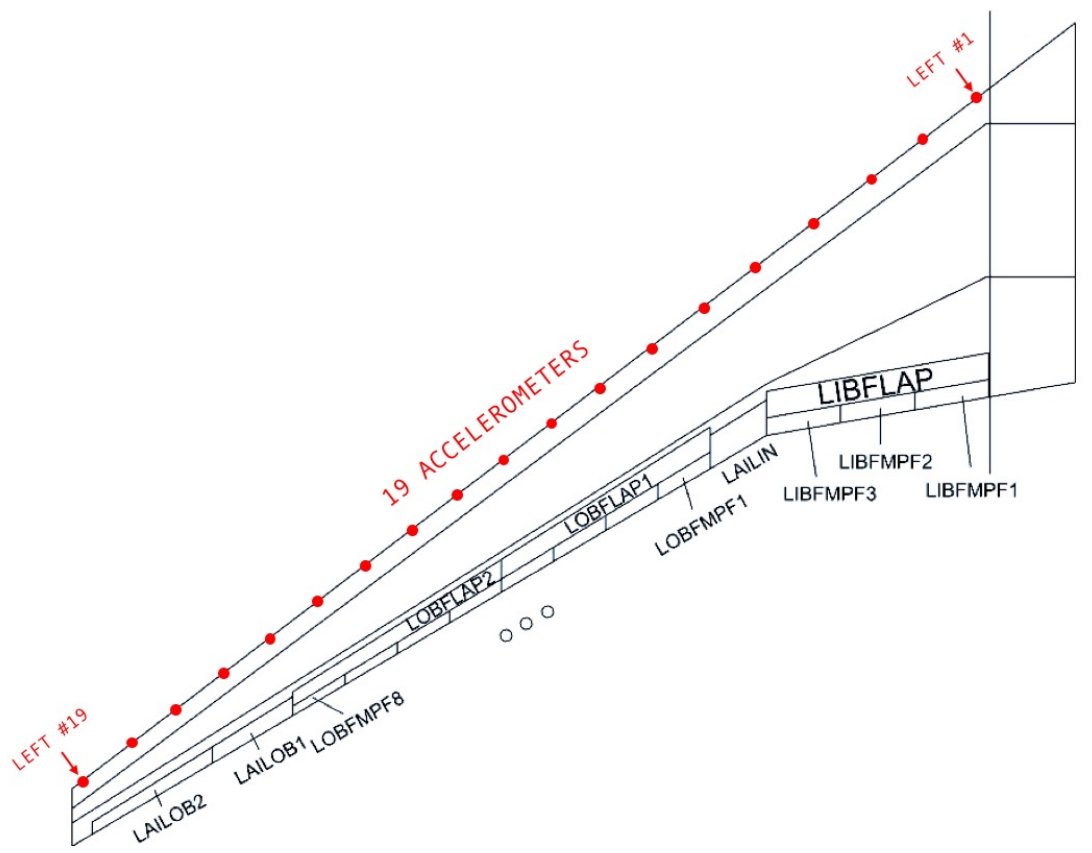

Figure 1. Trailing edge control surfaces and accelerometer locations for the CRM-13.5 model (Not to scale).

\section{Tracking Control}

With 17 control surfaces per wing in the full 6-DoF Blended Model, there are many reasonable strategies available for allocating control for different objectives. In this work, the two outer ailerons (R/LAILOB 1-2 in Fig. 1) and the rudder are used for lateral tracking control, and the elevator is used for longitudinal control. Control of the four ailerons is structured so that a 1 degree aileron command deflects both right ailerons 1 degree upward, and both left ailerons 1 degree downward, resulting in rightward roll of the aircraft. The eight mini-flaps (R/LOBFMPF 1-8) are utilized for multi-objective control detailed in the next section. The tracking controller involves both classical control methods and two LQR controllers with rigid body state feedback.

\section{A. Rigid Body LQR Tracking Control Development}

The goal of the nominal tracking controller is to track a desired flight path angle $\gamma_{c}$ in the longitudinal dimension, and heading angle $\psi_{c}$ in the lateral direction. It uses three primary command inputs: ailerons, elevator, and rudder, denoted $u_{t}=\left[\delta_{a}, \delta_{e}, \delta_{r}\right]^{T}$. Control is achieved by using full rigid body state feedback, integral and feedforward control. Gains are calculated separately using the two individual simple rigid body 3 -DoF lateral and longitudinal models mentioned above, but the resulting control design is to be applied to the full 6-DoF model. These simple rigid body models use state variables defined as

$$
x_{l a t}=\left[\begin{array}{lllll}
\beta & p & r & \phi & \psi
\end{array}\right]^{T}, \quad x_{l o n}=\left[\begin{array}{llll}
v_{t} & \alpha & q & \theta
\end{array}\right]^{T} .
$$

In the lateral direction, a coordinated turn filter is used to convert heading angle tracking error to a desired roll angle $\phi_{c}$, which is then tracked accordingly with aileron input $\delta_{a}$. A yaw damper with a washout filter is employed to command the rudder $\delta_{r}$. In the longitudinal direction, flight path angle is tracked with the elevator $\delta_{e}$. A description of these three primary tracking control laws follows.

\section{Roll Control}

The 3-DoF lateral model provided has a rudder input and 4 aileron inputs ( 2 per wing.) The aileron inputs are reduced to use only one input that counter activates the outer two ailerons on both wings such that 
positive input results in a rightward roll. This is identical to how the full sized 6-DoF model aileron control is allocated. Roll tracking control using the aileron input $\delta_{a}$ is defined as

$$
u_{\text {lat }}=\delta_{a}=K_{\phi e} \underbrace{\int\left(\phi-\phi_{c}\right) d \tau}_{e_{\phi}}+K_{\phi} x_{l a t}+K_{\phi f} \phi_{c} .
$$

The commanded roll $\phi_{c}$ is derived from the commanded heading angle $\psi_{c}$ via an outer loop consisting of a coordinated turn gain and filter. The coordinated turn controller attempts to roll the aircraft at the appropriate angle such that the vector sum of the centrifugal force and weight lines up with the vertical body axis of the aircraft. This requires that

$$
\tan \phi_{c}=\frac{v_{t} \dot{\psi}}{g}
$$

but with $\phi_{c} \ll 1$, this can be approximated as

$$
\phi_{c} \approx \frac{v_{t} \dot{\psi}}{g}
$$

Since $\dot{\psi}$ tends to be a noisy signal in implementation, it is filtered by

$$
\dot{\psi}=\frac{1}{\tau_{c}}\left(\psi_{c}-\psi\right)
$$

which provides a convenient relationship between commanded heading and roll yielding

$$
\phi_{c}=G_{c}\left(\psi_{c}-\psi\right)
$$

where $G_{c}=\frac{v_{t}}{\tau_{c} g}$.

The feedback and feedforward gains of Eq. 2 are calculated using the standard servomechanism LQR method using the extended state lateral model defined as

$$
\frac{d}{d t}\left[\begin{array}{c}
e_{\phi} \\
x_{\text {lat }}
\end{array}\right]=\left[\begin{array}{cc}
0 & C_{\phi} \\
0 & A_{\text {lat }}
\end{array}\right]\left[\begin{array}{c}
e_{\phi} \\
x_{\text {lat }}
\end{array}\right]+\left[\begin{array}{c}
D_{\phi} \\
B_{\text {lat }}
\end{array}\right] \delta_{a}+\left[\begin{array}{c}
-1 \\
0
\end{array}\right] \phi_{c} .
$$

\section{Flight Path Control}

The 3-DoF longitudinal model for this simulation uses the elevator input to track a commanded flight path angle $\gamma_{c}$. Similar to the roll control arrangement above, the control law for this model is

$$
u_{\text {lon }}=\delta_{e}=K_{\gamma e} \underbrace{\int\left(\gamma-\gamma_{c}\right) d \tau}_{e_{\gamma}}+K_{\gamma} x_{l o n}+K_{\gamma f} \gamma_{c},
$$

where the gains are again calculated using the servomechanism LQR method on the extended longitudinal state space model

$$
\frac{d}{d t}\left[\begin{array}{c}
e_{\gamma} \\
x_{l o n}
\end{array}\right]=\left[\begin{array}{cc}
0 & C_{\gamma} \\
0 & A_{\text {lon }}
\end{array}\right]\left[\begin{array}{c}
e_{\gamma} \\
x_{l o n}
\end{array}\right]+\left[\begin{array}{c}
D_{\gamma} \\
B_{l o n}
\end{array}\right] \delta_{e}+\left[\begin{array}{c}
-1 \\
0
\end{array}\right] \gamma_{c} .
$$

\section{Yaw Control}

A yaw damper is constructed to stabilize the Dutch Roll mode using a high-pass filter $H_{w}(s)$ in the feedback loop (known as a washout filter) so that slow yaw (heading) changes are permitted without being canceled out by the feedback loop. The yaw damper applies input to the rudder $\left(\delta_{r}\right)$ based on yaw rate $r$. The simple controller is defined as

$$
\delta_{r}=k_{w}\left(r_{c}-H_{w}(s) r\right) .
$$

Here, our commanded yaw rate $r_{c}$ is set to zero. Since the transfer function from $\delta_{r} \rightarrow r$ has negative gain, the washout gain $k_{w}$ is set to be negative. The washout filter is defined as

$$
H_{w}(s)=\frac{\tau_{w} s}{\tau_{w} s+1} .
$$

$$
4 \text { of } 21
$$




\section{B. Combined 6-DoF Control on the Blended Model}

Before proceeding to the multi-objective controller development, it is convenient to pose the tracking controller in a compact form applicable to the full 6 -DoF model so that it can be utilized in the multi-objective controller derivation.

The rigid body states of the blended model are defined as

$$
x_{6 \operatorname{dof}_{r b}}=\left[\begin{array}{lllllllll}
u & v & w & p & q & r & \phi & \theta & \psi
\end{array}\right]^{T}
$$

and as such do not match those used by the individual 3-DoF models. Fortunately, using provided outputs from the 6-DoF rigid body model for the missing states allows us to define a transformation matrix $\mathrm{T}$ such that

$$
\left[\begin{array}{l}
x_{l a t} \\
x_{l o n}
\end{array}\right]=\mathrm{T} x_{6 d o f_{r b}}
$$

Now the state feedback gains calculated above can be transformed to work on the states of the blended model in the appropriate order:

$$
\begin{aligned}
\bar{K}_{\phi} & =\left[\begin{array}{ll}
K_{\phi} & 0
\end{array}\right] \mathrm{T} \\
\bar{K}_{\gamma} & =\left[\begin{array}{ll}
0 & K_{\gamma}
\end{array}\right] \mathrm{T} .
\end{aligned}
$$

Note that the dimensions of both resulting gains are $1 \times 9$ since they are applied to the rigid body states only. The 6 -DofF model may be defined in state space form as

$$
\begin{aligned}
& \dot{x}=A x+B_{t} u_{t}+B_{m} u_{m} \\
& y=C x+D_{t} u_{t}+D_{m} u_{m}
\end{aligned}
$$

where the subscripts $t$ and $m$ indicate the tracking and mulit-objective control inputs respectively. Pertinent tracking control model outputs are roll, heading, flight path angle, and yaw rate, which are given respectively by

$$
\begin{aligned}
\phi & =C_{\phi} x+D_{\phi, t} u_{t}+D_{\phi, m} u_{m} \\
\psi & =C_{\psi} x+D_{\psi, t} u_{t}+D_{\psi, m} u_{m} \\
\gamma & =C_{\gamma} x+D_{\gamma, t} u_{t}+D_{\gamma, m} u_{m} \\
r & =C_{r} x+D_{r, t} u_{t}+D_{r, m} u_{m}
\end{aligned}
$$

The state space model for the washout filter $H_{w}(s)$ is defined as

$$
\begin{aligned}
& \dot{x}_{w}=A_{w} x_{w}+B_{w} r \\
& y_{w}=C_{w} x_{w}+D_{w} r .
\end{aligned}
$$

Combining all system dynamics up this point yields

$$
\begin{aligned}
\frac{d}{d t} \underbrace{\left[\begin{array}{c}
e_{\phi} \\
e_{\gamma} \\
x \\
x_{w}
\end{array}\right]}_{\boldsymbol{x}}=\underbrace{\left[\begin{array}{cccc}
0 & 0 & C_{\phi}+G_{c} C_{\psi} & 0 \\
0 & 0 & C_{\gamma} & 0 \\
0 & 0 & A & 0 \\
0 & 0 & B_{w} C_{r} & A_{w}
\end{array}\right]}_{\boldsymbol{A}_{\boldsymbol{o l}}}\left[\begin{array}{c}
e_{\phi} \\
e_{\gamma} \\
x \\
x_{w}
\end{array}\right]+\underbrace{\left[\begin{array}{c}
D_{\phi, t}+G_{c} D_{\psi, t} \\
D_{\gamma, t} \\
B_{t} \\
B_{w} D_{r, t}
\end{array}\right]}_{\boldsymbol{B}_{t}} u_{t}+ \\
\underbrace{\left[\begin{array}{c}
D_{\phi, f}+G_{c} D_{\psi, f} \\
D_{\gamma, f} \\
B_{f} \\
B_{w} D_{r, f}
\end{array}\right]}_{\boldsymbol{B}_{m}} u_{m}+\underbrace{\left[\begin{array}{cc}
-G_{c} & 0 \\
0 & -1 \\
0 & 0 \\
0 & 0
\end{array}\right]}_{\boldsymbol{B}_{r}}\left[\begin{array}{l}
\psi_{c} \\
\gamma_{c}
\end{array}\right] .
\end{aligned}
$$


Placed into this structure, the tracking control law can be written as

$$
u_{t}=\left[\begin{array}{c}
\delta_{a} \\
\delta_{e} \\
\delta_{r}
\end{array}\right]=\underbrace{\left[\begin{array}{cccc}
K_{\phi e} & 0 & {\left[\begin{array}{cc}
\bar{K}_{\phi} & 0
\end{array}\right]-K_{\phi f} G_{c} C_{\psi}} & 0 \\
0 & K_{\gamma e} & {\left[\begin{array}{cc}
\bar{K}_{\gamma} & 0
\end{array}\right]} & 0 \\
0 & 0 & -k_{w} D_{w} C_{r} & -k_{w} C_{w}
\end{array}\right]}_{\boldsymbol{K}_{t}} \boldsymbol{x}+\underbrace{\left[\begin{array}{cc}
G_{c} K_{\phi f} & 0 \\
0 & K_{\gamma f} \\
0 & 0
\end{array}\right]}_{\boldsymbol{K}_{f f}} \underbrace{\left[\begin{array}{c}
\psi_{c} \\
\gamma_{c}
\end{array}\right]}_{r} .
$$

The above tracking control law of Eq. 20 is defined and dimensioned accordingly using the extended state vector $\boldsymbol{x}$, which includes all plant states, integral error states $e_{\phi}$ and $e_{\gamma}$, and the washout filter state $x_{w}$. However, of all the plant states, only the rigid body states are utilized. Simulations shown in the Results section will assume perfect knowledge of these states, whereas states utilized by the multi-objective controller will be estimated.

\section{Multi-Objective Optimal Controller}

Using multi-objective optimal control, the full potential of PAAW technology may be realized. By selectively and/or simultaneously reducing the effects of structural modes/flutter, wing root bending, gust, and drag loads, multi-objective control has been successfully applied in varying forms to the longitudinal Generic Transport Model (GTM). ${ }^{2,6,7}$ This work continues herein applied to the CRM-13.5 model, and a time-varying version of muti-objective control is detailed in Ref. 8.

\section{A. Controller Derivation}

The multi-objective controller developed here is tasked with suppressing structural modes, reducing wing root bending moment, and reducing drag. It considers the effects of gust disturbances, so the aircraft plant model becomes

$$
\begin{aligned}
& \dot{x}=A x+B_{t} u_{t}+B_{m} u_{m}+B_{d} d \\
& y=C x+D_{t} u_{t}+D_{m} u_{m}+D_{d} d
\end{aligned}
$$

where $d$ is a 2-dimensional gust disturbance consisting of vertical gust velocity and acceleration. Incorporating the above tracking controller, and hereafter denoting the non-boldface $x$ to include all pertinent system and plant states, the closed loop dynamics are

$$
\dot{x}=\underbrace{\left(A_{o l}+B_{t} K_{t}\right)}_{\bar{A}} x+\underbrace{\left(B_{r}+B_{t} K_{f f}\right)}_{\bar{B}_{r}} r+B_{m} u_{m}+B_{d} d .
$$

Wing root bending moment $M_{y}$ is defined as

$$
M_{y}=\bar{M}_{x} x+\bar{M}_{r} r+M_{u_{m}} u_{m}+M_{d} d
$$

where

$$
\begin{aligned}
\bar{M}_{x} & =M_{x}+M_{u_{t}} K_{t} \\
\bar{M}_{r} & =M_{u_{t}} K_{f f}
\end{aligned}
$$

The drag model was not provided by Boeing, but was developed based on a perturbed model about the trim condition. With closed loop tracking control, it can be defined as

$$
\Delta C_{D}=\bar{C}_{D_{x}} x+\bar{C}_{D_{r}} r+x^{T} \bar{C}_{D_{x^{2}}} x+r^{T} \bar{C}_{D_{r x}} x+C_{D_{u_{m}}} u_{m}+C_{D_{d}} d+x^{T} C_{D_{x d}} d+x^{T} C_{D_{x u_{m}}} u_{m} .
$$

where

$$
\begin{aligned}
\bar{C}_{D_{x}} & =C_{D_{x}}+C_{D_{u_{n}}} K_{t} \\
\bar{C}_{D_{r}} & =C_{D_{u_{n}}} K_{f f} \\
\bar{C}_{D_{x^{2}}} & =C_{D_{x^{2}}}+C_{D_{u_{n}}} K_{t} \\
\bar{C}_{D_{r x}} & =\left(C_{D_{u_{n}}} K_{f f}\right)^{T} .
\end{aligned}
$$


The multi-objective controller is defined as

$$
u_{m}=K_{x} \hat{x}+K_{r} r+K_{d} \hat{d}+\Lambda .
$$

State and gust estimates $\hat{x}$ and $\hat{d}$ can be provided using several different strategies. A discussion of how they are generated for this work is provided in the next section.

The above control law is based on the cost function

$$
J=\lim _{t_{f} \rightarrow \infty} \int_{0}^{t_{f}}\left(x^{T} Q x+u_{m}^{T} R u_{m}+q_{M} M_{y}^{2}+q_{D} \Delta C_{D}\right) d t
$$

where $Q$ is the state suppression weights, $R$ multi-objective control weights, $q_{M}$ and $q_{D}$ the wing root bending moment and drag reduction weights respectively.

The gains from Eq. 31 are functions of $W$, which is the solution to the algebraic Riccati equation

$$
W \overline{\bar{A}}+\overline{\bar{A}}^{T} W-W B_{m} \bar{R}^{-1} B_{m} W+\bar{Q}=0
$$

where

$$
\begin{gathered}
\bar{R}=R+q_{M} M_{u_{m}}^{T} M_{u_{m}} \\
\overline{\bar{A}}=\bar{A}-\frac{1}{2} q_{D} B_{m} \bar{R}^{-1} C_{D_{x u_{m}}}^{T}-q_{M} B_{m} \bar{R}^{-1} M_{u_{m}}^{T} \bar{M}_{x} \\
\bar{Q}=Q+q_{D} \bar{C}_{D_{x^{2}}}+q_{M} \bar{M}_{x}^{T} \bar{M}_{x} \\
-\left(\frac{1}{2} q_{D} C_{D_{x u_{m}}}+q_{M} \bar{M}_{x}^{T} M_{u_{m}}\right) \bar{R}^{-1}\left(\frac{1}{2} q_{D} C_{D_{x u_{m}}}^{T}+q_{M} M_{u_{m}}^{T} \bar{M}_{x}\right) .
\end{gathered}
$$

Given the following definitions,

$$
\begin{aligned}
& \overline{\bar{V}}=\overline{\bar{A}}^{T}-W B_{m} \bar{R}^{-1} B_{m} \\
& V_{r}=-\overline{\bar{V}}^{-1}\left[W \bar{B}_{r}+\frac{1}{2} q_{D} \bar{C}_{D_{r x}}^{T}+q_{M} \bar{M}_{x}^{T} \bar{M}_{r}-W B_{m} \bar{R}^{-1}\left(q_{M} M_{u_{m}}^{T} \bar{M}_{r}\right)\right. \\
& \left.-\left(\frac{1}{2} q_{D} C_{D_{x u_{m}}}+q_{M} \bar{M}_{x}^{T} M_{u_{m}}\right) \bar{R}^{-1}\left(q_{M} M_{u_{m}}^{T} \bar{M}_{r}\right)\right] \\
& V_{d}=-\overline{\bar{V}}^{-1}\left[W B_{g}+\frac{1}{2} q_{D} \bar{C}_{D_{x d}}+q_{M} \bar{M}_{x}^{T} \bar{M}_{d}-W B_{m} \bar{R}^{-1}\left(q_{M} M_{u_{m}}^{T} \bar{M}_{d}\right)\right. \\
& \left.-\left(\frac{1}{2} q_{D} C_{D_{x u_{m}}}+q_{M} \bar{M}_{x}^{T} M_{u_{m}}\right) \bar{R}^{-1}\left(q_{M} M_{u_{m}}^{T} M_{d}\right)\right] \\
& V_{0}=-\overline{\bar{V}}^{-1}\left[\frac{1}{2} q_{D} \bar{C}_{D_{x}}^{T}-W B_{m} \bar{R}^{-1}\left(\frac{1}{2} q_{D} C_{D_{u_{m}}}^{T}\right)\right. \\
& \left.-\left(\frac{1}{2} q_{D} C_{D_{x u_{m}}}+q_{M} \bar{M}_{x}^{T} M_{u_{m}}\right) \bar{R}^{-1}\left(\frac{1}{2} q_{D} C_{D_{u_{m}}}^{T}\right)\right]
\end{aligned}
$$

the gains from Eq. 31 are

$$
\begin{aligned}
K_{x} & =-\bar{R}^{-1}\left(\frac{1}{2} q_{D} C_{D_{x u_{m}}}^{T}+q_{M} M_{u_{m}}^{T} \bar{M}_{x}+B_{m}^{T} W\right) \\
K_{r} & =-\bar{R}^{-1}\left(q_{M} M_{u_{m}}^{T} \bar{M}_{r}+B_{m}^{T} V_{r}\right) \\
K_{d} & =-\bar{R}^{-1}\left(q_{M} M_{u_{m}}^{T} M_{d}+B_{m}^{T} V_{d}\right) \\
\Lambda & =-\bar{R}^{-1}\left(\frac{1}{2} q_{D} C_{D_{u_{m}}}^{T}+B_{m}^{T} V_{0}\right) .
\end{aligned}
$$

Since this multi-objective controller requires estimates for state and gust signals, the following section describes how those estimates are generated for the CRM-13.5 model.

\section{7 of 21}




\section{State and Gust Estimation}

Generating state estimates for the current CRM-13.5 model has been a challenging endeavor. Standard techniques of calculating optimal Kalman state estimator gains often result in warnings or errors from MATLAB's standard kalman command (or, equivalently, the lqr command applied to the dual system) due to poor observability/detectibility. Some success is achieved by selecting high weights for the structural mode portions of state vector, and/or by judicious selection of plant and observer outputs supplied to the observer. Also, with 491 states, having to integrate both the "true" plant, actuators, and observer can provide a challenge during simulation depending on the resulting closed loop system dynamics stiffness and size. Therefore, it is reasonable to investigate strategies of model reduction for observer and control gain calculations.

\section{A. Model Reduction}

Two standard techniques of model state reduction were investigated and applied to the CRM-13.5. The first involves removing unused and/or undesired states using the Singular Perturbation method. The other involves transforming the system into a modal form, then eliminating states above a selected frequency. Ultimately, a combination of the two methods will be shown to provide the best performance in Section VII.

\section{Specific State Elimination}

One method of model reduction is to identify specific states for removal. Many states can be removed prior to any other reduction method since they will have no coupling to the input/output behavior of the model. These include the 6 control lag states associated with each unused control surface, and the 8 gust lag states when gust is not simulated, Analysis of the model reveals that the 6 lag states associated with the $u$ rigid body state are uncoupled from all other states, and can be eliminated. Finally, note that the control lag states only come into use when the actuator model is used, since it provides inputs for control surface rate and rate-rate. If the actuator model is unused, all control lags can be eliminated.

Since the 27 structural modes are ordered from lowest to highest frequency, it is reasonable to explore eliminating some of the higher frequency modes. Alternatively, if a simulation is restricted to longitudinal (symmetric) dynamics, it is reasonable to eliminate antisymmetric modes and vice versa. For each mode that is eliminated, its mode position and rate states should be removed, along with its 6 associated lag states.

Once states have been selected for elimination, rather than simply truncating them, the so-called Singular Perturbation method is employed.

\section{Singular Perturbation Model Reduction}

Given a state space model

$$
\begin{aligned}
& \dot{x}=A x+B u \\
& y=C x+D u,
\end{aligned}
$$

and organizing into states that we wish to preserve $x_{1}$, and those we wish to eliminate $x_{2}$ gives

$$
\begin{aligned}
\frac{d}{d t}\left[\begin{array}{l}
x_{1} \\
x_{2}
\end{array}\right] & =\left[\begin{array}{ll}
A_{11} & A_{12} \\
A_{21} & A_{22}
\end{array}\right]\left[\begin{array}{l}
x_{1} \\
x_{2}
\end{array}\right]+\left[\begin{array}{l}
B_{1} \\
B_{2}
\end{array}\right] u \\
y & =\left[\begin{array}{ll}
C_{1} & C_{2}
\end{array}\right]\left[\begin{array}{l}
x_{1} \\
x_{2}
\end{array}\right]+D u .
\end{aligned}
$$

The Singular Perturbation method assumes that $\dot{x}_{2} \approx 0$, which gives

$$
x_{2}=-A_{22}^{-1}\left(A_{21} x_{1}+B_{2} u\right),
$$

and after substituting into Eq. 57, gives

$$
\begin{aligned}
\dot{x}_{1} & =\left[A_{11}-A_{12} A_{22}^{-1} A_{21}\right] x_{1}+\left[B_{1}-A_{12} A_{22}^{-1} B_{2}\right] u \\
y & =\left[C_{1}-C_{2} A_{22}^{-1} A_{21}\right] x_{1}+\left[D-C_{2} A_{22}^{-1} B_{2}\right] u .
\end{aligned}
$$


Note that the above reduction technique is utilized by MATLAB's "modred" function with the default "MatchDC" option.

\section{Modal Reduction}

Another common method of model reduction, which may be applied in conjunction with the previous strategy is to eliminate portions of the model with dynamics beyond a desired range. Note that the word "modal" here is in the generic sense, and does not refer to specific aeroelastic structural modes that are in the model. This is accomplished by applying a sorted eigenvector transformation to the system. We seek to eliminate some portion of the state vector through the use of the modal transformation $x=T z \rightarrow z=T^{-1} x$. Here $\Lambda=T^{-1} A T$ is the diagonal matrix of eigenvalues sorted from lowest frequency to highest, and $T$ is sorted accordingly. Then, $T$ and $\Lambda$ can be partitioned by

$$
T=\left[\begin{array}{ll}
T_{1} & T_{2}
\end{array}\right], \quad \Lambda=\left[\begin{array}{cc}
\Lambda_{1} & 0 \\
0 & \Lambda_{2}
\end{array}\right]
$$

and the transformed partitioned system becomes

$$
\begin{aligned}
\frac{d}{d t}\left[\begin{array}{l}
z_{1} \\
z_{2}
\end{array}\right] & =\left[\begin{array}{cc}
\Lambda_{1} & 0 \\
0 & \Lambda_{2}
\end{array}\right]\left[\begin{array}{l}
z_{1} \\
z_{2}
\end{array}\right]+\left[\begin{array}{l}
R_{1} \\
R_{2}
\end{array}\right] u \\
y & =\left[\begin{array}{ll}
C T_{1} & C T_{2}
\end{array}\right]\left[\begin{array}{l}
z_{1} \\
z_{2}
\end{array}\right]+D u
\end{aligned}
$$

where $R_{1}$ consists of the first $n_{1}$ rows of $T^{-1} B$, and $R_{2}$ consists of remaining rows. This system is then reduced to

$$
\begin{aligned}
\dot{z}_{1} & =\Lambda_{1} z_{1}+R_{1} u \\
y & =C T_{1} z_{1}+D u
\end{aligned}
$$

via simple truncation. Singular Perturbation is unnecessary since there are no off-diagonal terms in the state matrix.

\section{Model Reduction Comparison}

In order to briefly explore the accuracy and fidelity of applying these reduction techniques to the model, we present a simple simulation. We select case number 30 from the blended CRM models. This corresponds to a structurally unstable condition at Mach 0.85, zero fuel load and an altitude of 19,607 ft. The system consists of only the elevator input and the angle of attack for output. We attach the actuator model in order to provide rate and rate-rate inputs for the elevator dynamics. The various degrees of model reduction are described below.

1. Full System: The non reduced full system including all lags and all actuator states. $n=564$.

2. Baseline: This is the Full System without the unused actuator states, unused control surface lags, and unused gust lags. $n=269$.

3. Modes 1-20: This is the Baseline system but with only the first 20 modes and associated lags. The mode positions, mode velocities and 6 lag states associated with the last 7 modes have been removed via Singular Perturbation. $n=213$.

4. Symmetric Modes: The Baseline system but with only the symmetric modes. Antisymmetric modes and associated lags have been removed via Singular Perturbation. $n=173$.

5. Modal Reduction: $4 \mathbf{H z}$ Cutoff: The Baseline system with Modal Reduction. All poles of the entire system above a frequency of $4 \mathrm{~Hz}$ have been removed. $n=100$. 


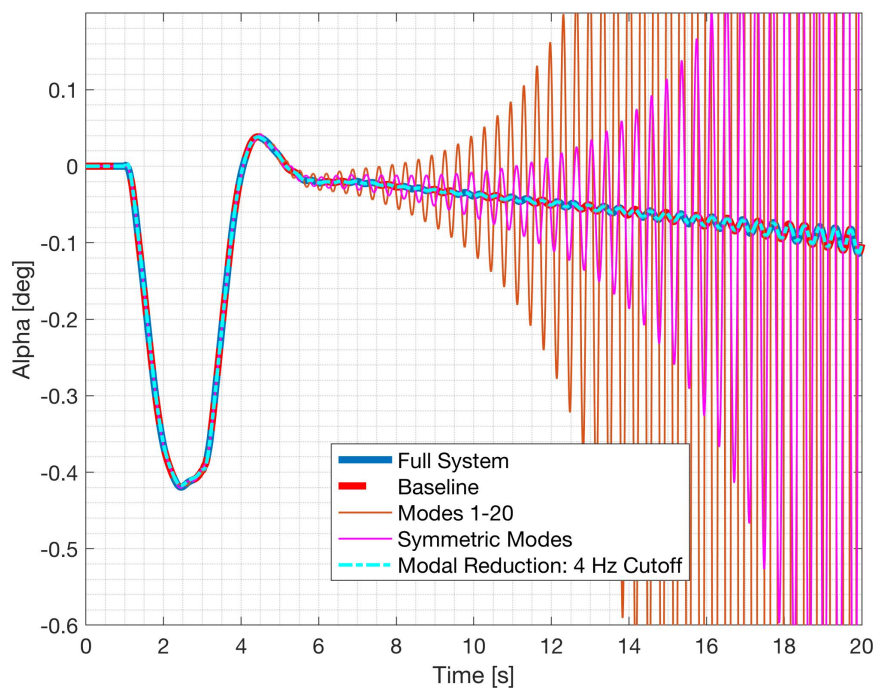

Figure 2. Time response of the actuator-plant model with various levels of reduction.

We simulate the systems with a 1 degree elevator deflection between 1 and 3 seconds. Results are shown in Fig. 2. Notice that all the systems respond similarly when responding to the elevator input, and eventually all the systems explode due to the open loop instability. However, the Modal Reduction technique with a 4 $\mathrm{Hz}$ cutoff (which is also the smallest model) is the most accurate. In fact, it is indistinguishable from the Baseline and Full System models.

Note that the unstable ringing at approximately $2.6 \mathrm{~Hz}$ is more accurate for the Modal Reduction system because this unstable pole is unchanged, whereas the state-specific reduction methods (e.g., Modes 1-20, and Symmetric Modes) alter the locations of all remaining poles when reduction occurs.

\section{Reduced Model for Estimation}

The Modal Reduction Version technique described above can be applied with the transformation matrix calculated from the eigenvector results of MATLAB's eig command. One drawback to this approach is that the resulting system can involve matrices with complex entries, which can complicate simulation. MATLAB includes a function called freqsep, which performs modal reduction on the model by eliminating all modes greater than a desired frequency, and results in real-valued state space matrices. The drawback to freqsep is that it does not provide the transformation matrix used in the modal reduction. Such a matrix can be useful to have, especially for mapping optimal LQG state weights for estimation and control from the original model to those in the reduced model. A work-around is to perform a modal transformation on the system using the canon command, which does return the transformation matrix $T$. After resorting $T$ according to eigenvalue magnitude, the system can be manually transformed with $T$ using standard techniques.

Assuming an LQG flutter suppression controller can be designed on the non-reduced system, it will typically have non-zero diagonal entries of the $Q$ matrix corresponding to the structural mode states. Defining $q$ as the diagonal entries of the non-reduced system's $Q$ matrix, we define $\bar{Q}$ as:

$$
\bar{Q}=\left[\begin{array}{lll}
\ddots & & 0 \\
& q & \\
0 & & \ddots
\end{array}\right]^{\frac{1}{2}} T_{1}
$$

which will have the dimension $n \times n_{\text {red }}$. The weighting matrix for the reduced system $Q_{r e d}$ is then defined as

$$
Q_{\text {red }}=\bar{Q}^{T} \bar{Q}
$$


Simulation results of the above reduction technique applied to LQG flutter suppression are shown in Section VII.

\section{B. Observer Implementation Reduction}

As mentioned above, the relatively large system size of this model can make practical simulation a difficult and slow process, especially with the addition of an observer. Thus, it is always desirable to reduce the number of states that require integration so long as fidelity is preserved.

Assuming that observer gains $L$ can be found, the observer can be described by

$$
\begin{array}{r}
\dot{\hat{z}}=A_{o} \hat{z}+B_{o}\left[\begin{array}{c}
u_{t} \\
u_{m}
\end{array}\right]+L(y-\hat{y}) \\
\hat{y}=C_{o} \hat{z}+D_{o}\left[\begin{array}{c}
u_{t} \\
u_{m}
\end{array}\right]
\end{array}
$$

where $\hat{z}$ consists of state and possibly disturbance estimates (described below). The above can be arranged into standard state space form as

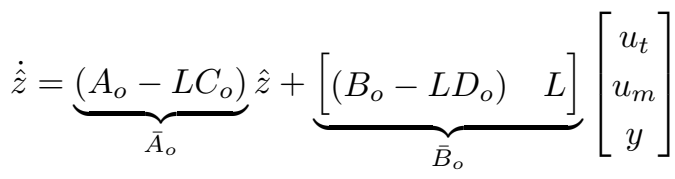

$$
\begin{aligned}
& \hat{z}=\underbrace{I}_{\bar{C}_{o}} \hat{z}+\underbrace{0}_{\bar{D}_{o}}\left[\begin{array}{c}
u_{t} \\
u_{m} \\
y
\end{array}\right] \text {. }
\end{aligned}
$$

Then, using a method like the one used by MATLAB's minreal function on the system $\left(\bar{A}_{o}, \bar{B}_{o}, \bar{C}_{o}, \bar{D}_{o}\right)$, the observer can often be reduced substantially due to the presence of pole/zero cancellations and linearly coupled estimated outputs $\hat{z}$. The observer is then implemented as a state space system with system inputs $u_{t}, u_{m}$, and $y$ as input, and the estimated states and gusts $\hat{z}$ as outputs, even if the number of internal observer states has been reduced. Note that the previous two model reduction techniques reduce the number of states that are used for estimation and control. Here, the number of estimated states is maintained, but the number of integrated states required for the estimates is potentially reduced. This method can be applied as an addition to the previous methods, and is shown to provide good results and significant computational savings in practice.

\section{Gust Estimation}

There are several known methods for estimating system disturbances, and in particular gust disturbance. Hashemi et al in Ref. 6 describes several gust estimation strategies in the context of the GTM. These include estimating the gust system matrices, adaptive gust estimation, and gust estimation using an extended state observer. Here, we employ the extended state observer.

Since the gust signal disturbance consists of vertical velocity and acceleration $[w \dot{w}]^{T}$, the extended state observer takes the form

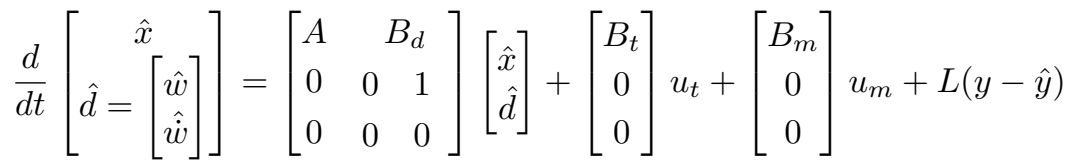

$$
\begin{aligned}
& \hat{y}=\left[\begin{array}{ll}
C & D_{d}
\end{array}\right]\left[\begin{array}{l}
\hat{x} \\
\hat{d}
\end{array}\right]+D_{t} u_{t}+D_{m} u_{m} .
\end{aligned}
$$

Thus, both state and gust estimates are achieved simultaneously. Note that the state estimate $\hat{x}$ from above may actually be a reduced state vector if model reduction is employed. 


\section{Results}

The above control structure is created and simulated in MATLAB/Simulink. The top level of the controller interconnections is shown in Fig. 3. The Aircraft model contains within it the full blended model and actuator model. Control lags and actuator states of unused control surfaces have been removed. The resulting plant contains 451 states. The actuator model states have been transformed to efficiently enforce deflection and rate limits of $\pm 25^{\circ}$, and $\pm 60^{\circ} \mathrm{s}^{-1}$ respectively. Anti-windup logic is employed to prevent erroneous behavior at the limit.

All simulations presented in this section were integrated using a fixed time step of $0.001 \mathrm{~s}$. Simulink was permitted to automatically select among its fixed-step integration methods. This resulted in the ode3 algorithm being selected, which is a variation of a third-order Runge-Kutta method known as the BogackiShampine Formula.

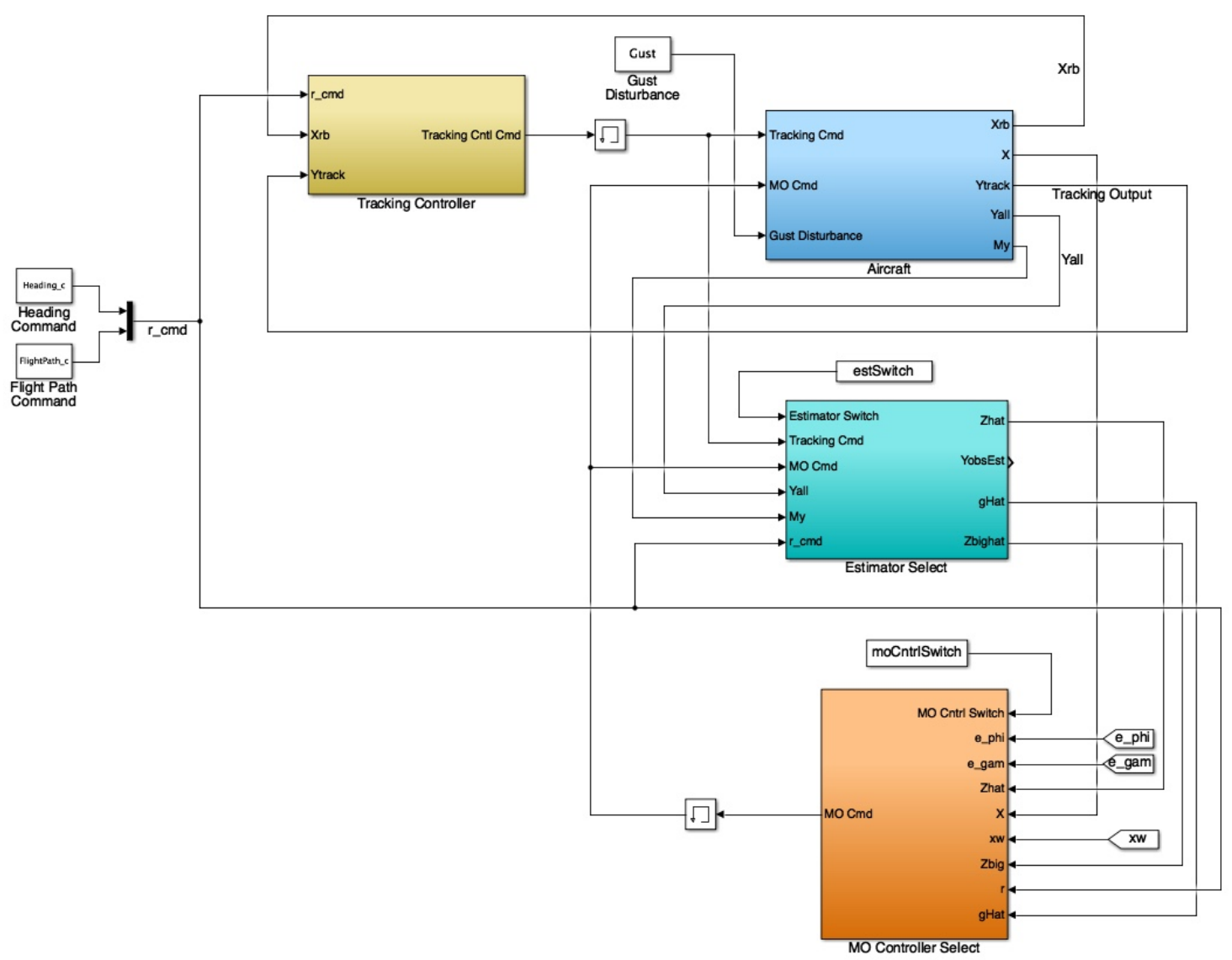

Figure 3. Top level view of the Simulink model showing tracking control, aircraft model, estimator, and multi-objective controller.

\section{A. Tracking Control at Cruise}

We begin with a demonstration of the 6-DoF tracking controller. The signal diagram for the tracking controller of Eq. 20 is shown in Fig. 4. Recall that this is an LQR controller derived from the separate simple rigid body models with servomechanism control for tracking. Thus, both feedback, which operates on perfect knowledge of the rigid body states, and feedforward gains are present. 


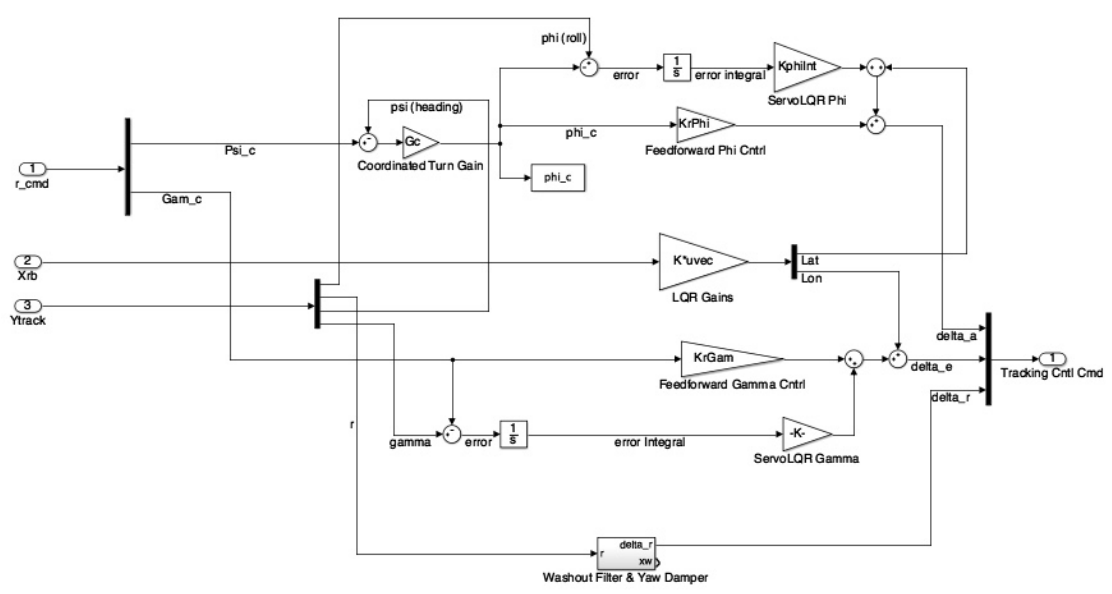

Figure 4. Tracking controller model.

For the first simulation, a trim model is selected matching a cruise condition having a speed of Mach $=$ 0.85 , altitude $=36,983 \mathrm{ft}$, and a full fuel load with a gross vehicle weight of 581,497 lb. A flight path angle doublet command is issued at the beginning of the simulation, followed by a 15 degree change in heading command. The results are shown in Figs. 5 and 6 along with their corresponding control surface commanded (shown dashed) and actual deflections. Note that in tracking the commanded heading $\psi_{c}$, a commanded roll signal $\phi_{c}$ is produced from the coordinated turn relation of Eq. 6. Also, note that the first most-inboard aileron deflections are not visible on the plot because they are identical to the outboard deflections due to the control allocation scheme.
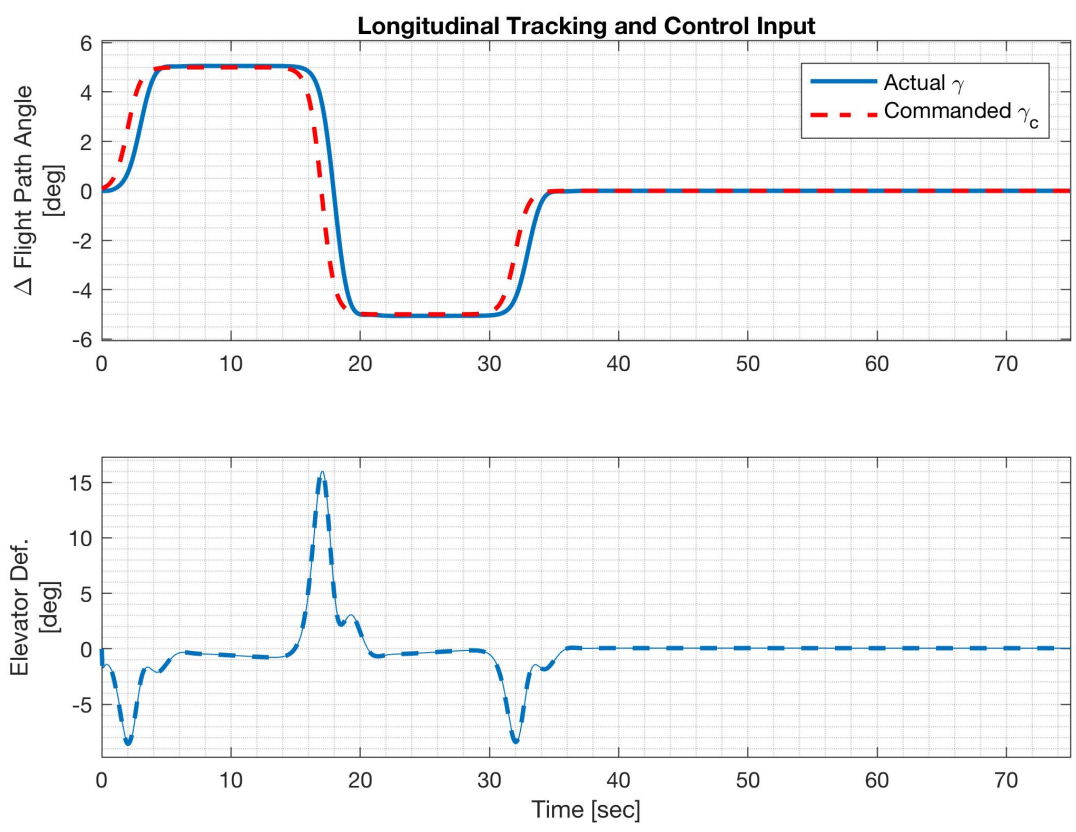

Figure 5. Longitudinal Tracking and Control Input at a cruise condition of Mach $=0.85$, altitude $=36,983$ ft, and full fuel load. 

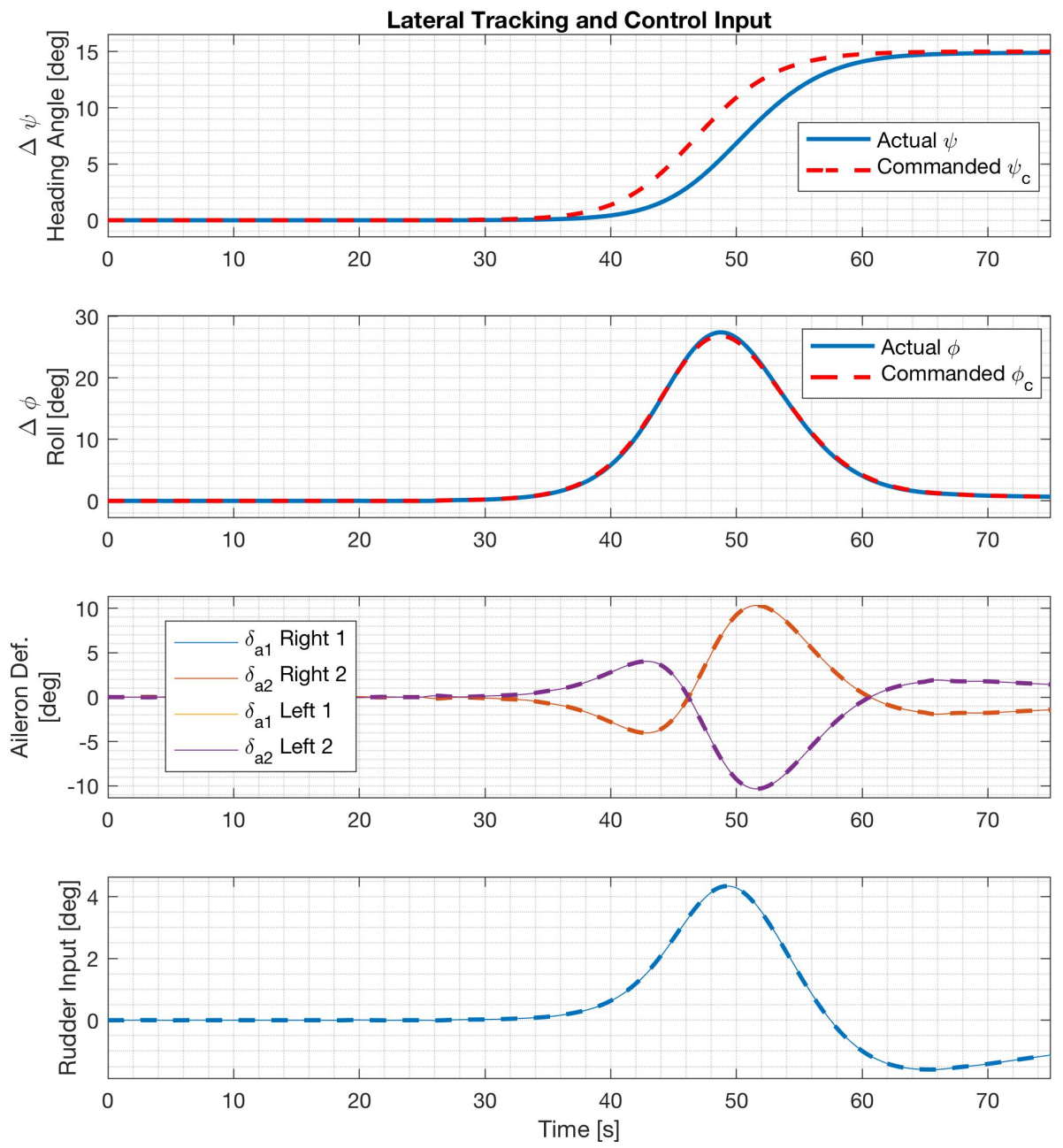

Figure 6. Lateral Tracking and Control Input at a cruise condition of Mach $=0.85$, altitude $=36,983 \mathrm{ft}$, and full fuel load.

\section{B. Unstable Flutter Suppression}

Prior to presenting the results of the multi-objective controller, it is worth investigating the estimation method shown in Section VI 4. Here, we look at the performance of an LQG regulator used to suppress an unstable flutter condition.

Of the 60 trim condition models provided by Boeing, several have been identified as being beyond the flutter boundary. At Mach $=0.85$, an altitude of 19,607 ft, and a gross vehicle weight of 383,546 lb (corresponding to an empty fuel condition), the aircraft model contains two unstable complex poles at $0.220 \pm 15.85 i$ marked with a blue $\times$ in Fig. 7 . 


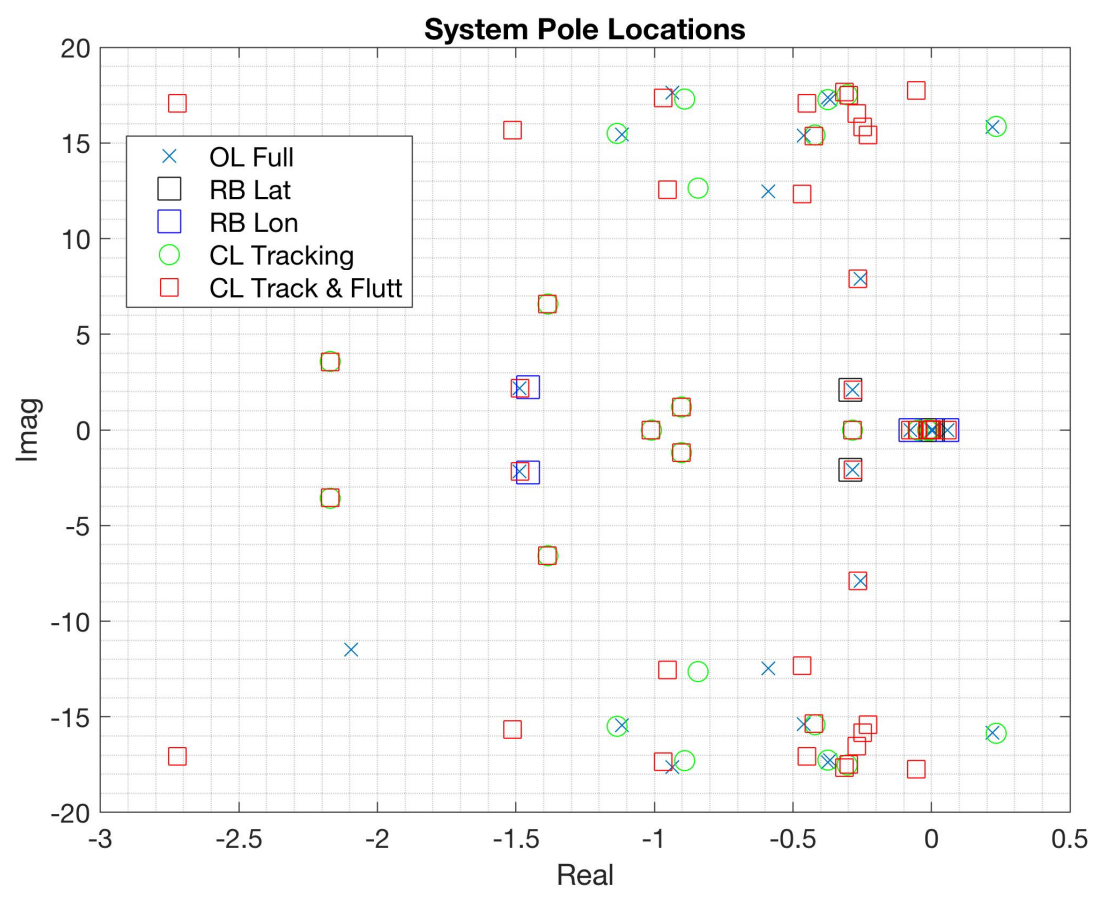

Figure 7. Open and closed system poles at a trim condition of Mach $=0.85$, altitude $=19,607 \mathrm{ft}$., and empty fuel condition. (Not all poles shown.)

Closing the loop with only tracking control does not significantly move these unstable poles (marked by the green circles $\circ$ ). This is demonstrated in a flight path angle tracking simulation. Results are initially very similar to those of Fig. 5 above. However, Fig. 8 reveals a $2.5 \mathrm{~Hz}$ (matching the frequency of the two unstable poles) unstable flutter response observed by accelerometers at the wing tips (left and right 19).

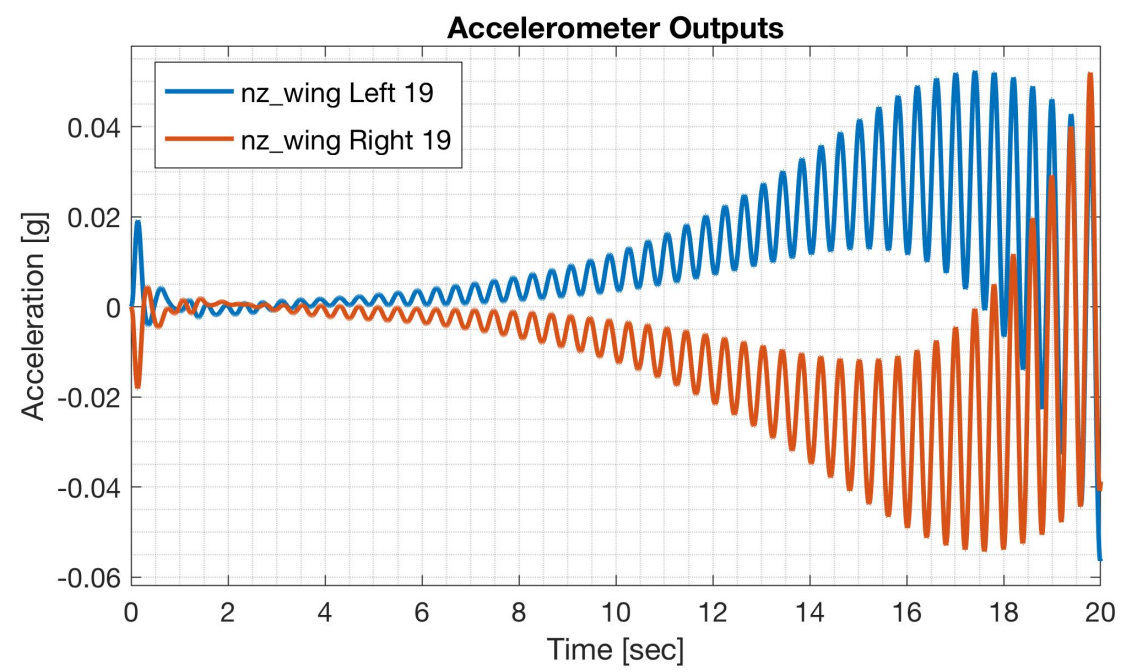

Figure 8. Unstable flutter response detected by wing accelerometers at a trim condition of $\mathrm{Mach}=0.85$, altitude $=19,607 \mathrm{ft}$., and empty fuel condition.

Next, the flutter suppression controller is implemented using the eight miniflaps (indicated for the left wing as flaps LOBFMPF1-8 in Fig. 1). The flutter suppression control law is given by

$$
u_{m}=K_{\text {flut }} \hat{z}
$$


where $\hat{z}$ is an estimated reduced state vector given by the observer

$$
\begin{aligned}
\dot{\hat{z}} & =A_{\text {red }} \hat{z}+B_{\text {red }_{t}} u_{t}+B_{\text {red }_{m}} u_{m}+L\left(y_{\text {obs }}-\hat{y}_{\text {obs }}\right) \\
\hat{y}_{\text {obs }} & =C_{\text {red }} \hat{z}+D_{\text {red }_{t}} u_{t}+D_{\text {red }_{m}} u_{m} .
\end{aligned}
$$

The observer system $\left(A_{r e d},\left[B_{r e d_{t}}, B_{r e d_{m}}\right], C_{r e d},\left[D_{r e d_{t}}, D_{r e d_{m}}\right]\right)$ is created by first removing unused control lag, and gust states, resulting in a system with 255 states. Actuator dynamics are ignored (but present in the aircraft plant during simulation). Next, the model is further reduced to 64 states using the modal reduction technique discussed above with a $3 \mathrm{~Hz}$ cutoff frequency. State weights used for calculating $L$ using the Kalman method are concentrated on the original system's structural mode position and velocity states, then transformed by the desired portion of the modal transformation matrix $T_{1}$. The observer uses plant outputs $y_{\text {obs }}$ consisting of $\phi, \psi, \gamma$, and the two wing tip accelerometers. $K_{f l u t}$ is calculated using $\left(A_{r e d}, B r e d_{m}\right)$.

The resulting full closed loop system is capable of stabilizing the flutter mode, as indicated by the red square $\square$ pole locations marked in Fig. 7. Simulating the system with the addition of flutter control and the same commanded flight path as before, the tracking results are nearly identical, but the accelerometers display stable wing movement as shown in Fig. 9. Thus, even with a significantly reduced observer, the flutter suppression controller performs as expected producing a stable closed loop system. Note the symmetric control response between the right and left wings, indicating that the unstable flutter mode is a symmetric mode.
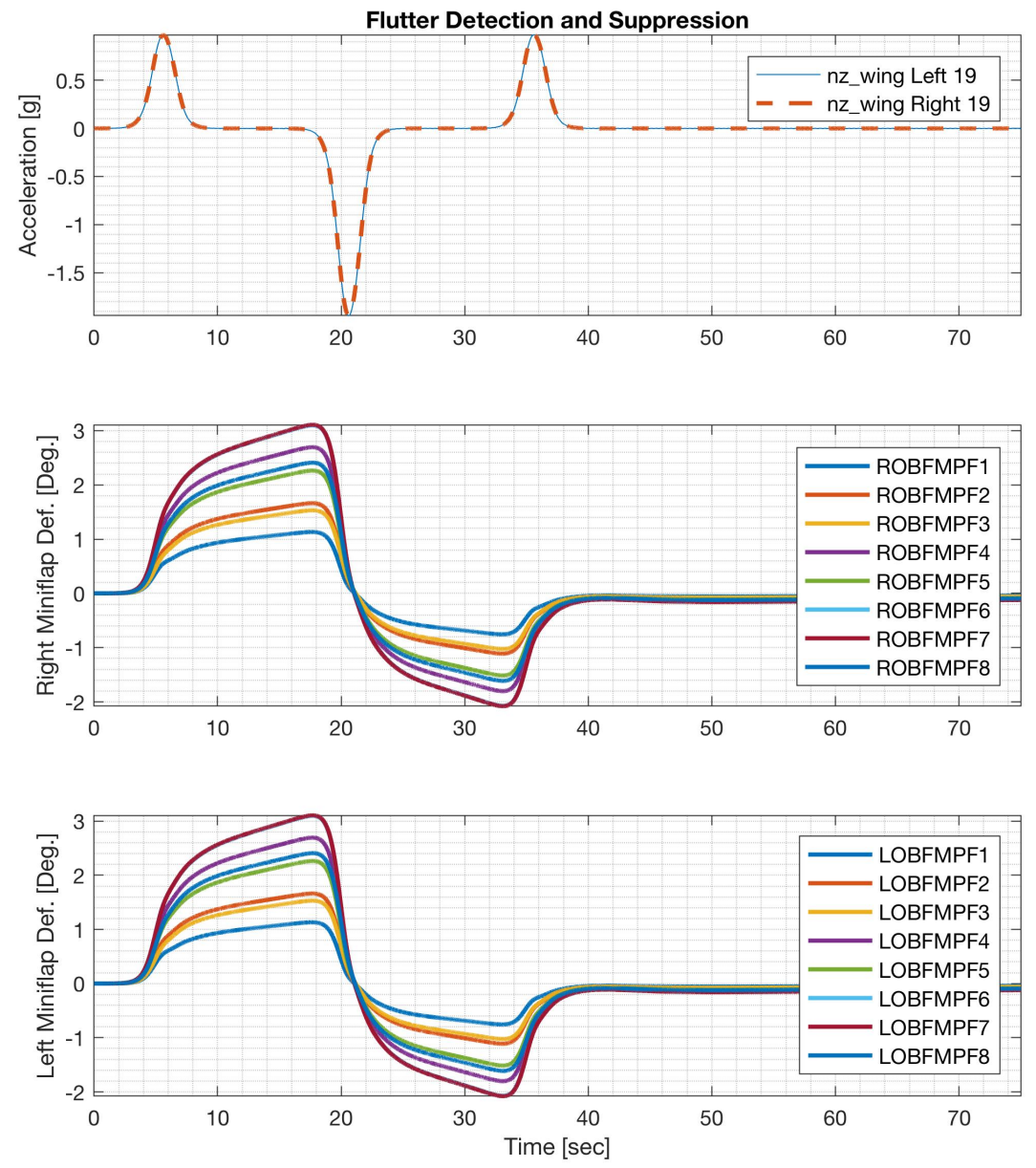

Figure 9. Stabilized flutter response wing acceleration and flutter suppression flap action. Trim condition of Mach $=0.85$, altitude $=19,607 \mathrm{ft}$., and empty fuel condition. 


\section{Multi-Objective Control}

In this section, we present the results of the multi-objective controller. We use the same cruise level flight condition selected for the first simulation, however there is an added 1-cosine gust disturbance at the 70 second mark lasting for 25 seconds. The tracking results are shown in Fig. 10. Note how the gust disturbance negatively affects the flight path angle.
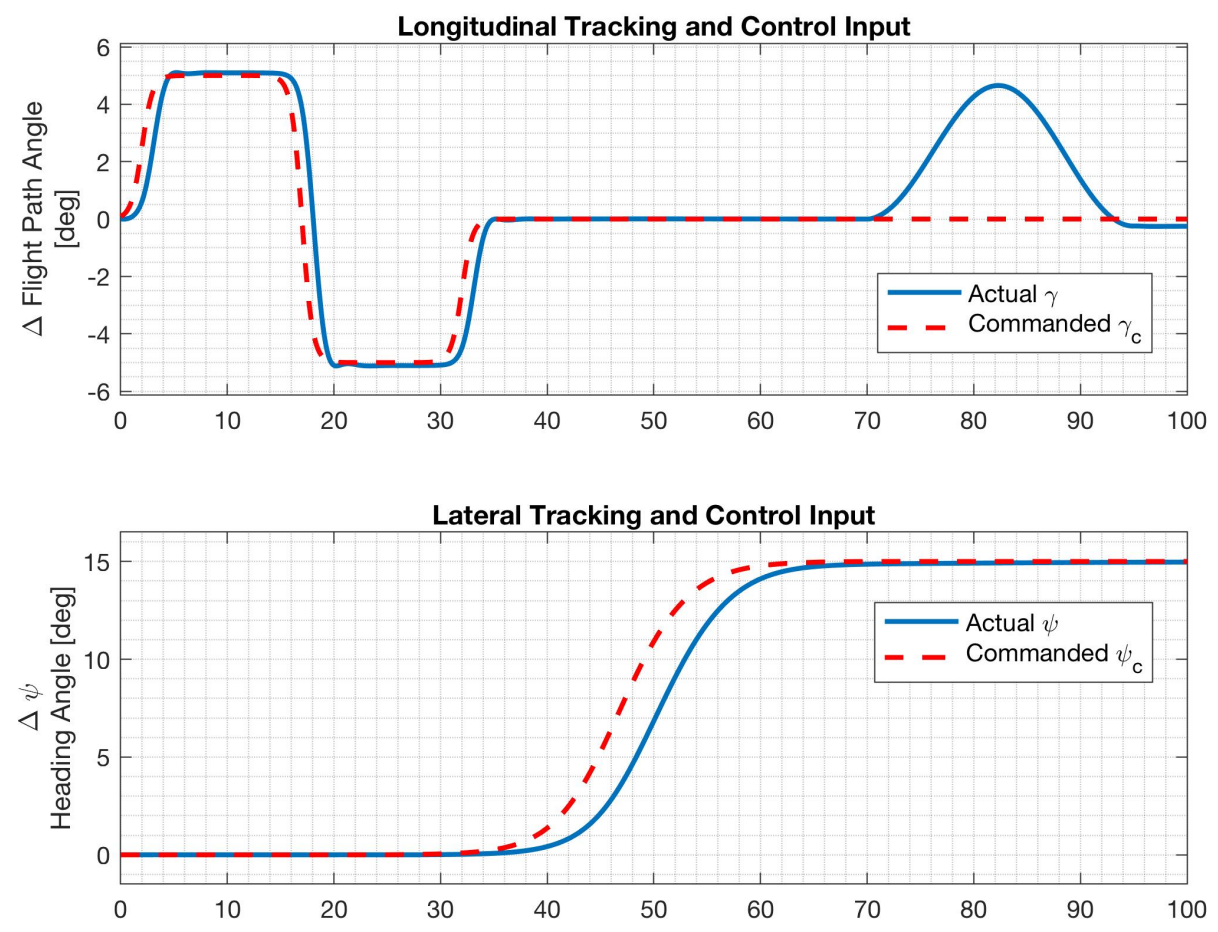

Figure 10. Longitudinal and Lateral Tracking Control at a cruise condition of Mach $=0.85$, altitude $=36,983$ $\mathrm{ft}$, and full fuel load.

Recall that the multi-objective controller of Eq. 31 requires both state and gust disturbance estimates. The extended state observer of Section VI C is used for this purpose. Selecting outputs and weights that resulted in a solution for the observer gains $L$ and produced a good gust estimate was a painstaking task. Unlike the LQG flutter suppression case presented earlier, it was found that neither specific state removal nor modal model reduction produced adequately accurate gust estimates. Thus the full model (minus the unused control surface lags) was used consisting of 407 states. However 138 states of were eliminated for simulation using the technique described in Section VI B, saving approximately 10 seconds of computation time for the simulation.

It was found that five plant outputs consisting of 3 rigid body outputs $(\phi, r, \psi)$ and the two wing tip accelerometers resulted in satisfactory estimates. The performance of the extended state observer is shown in Fig. 11. Gust estimation is shown to be quite accurate, with only minor errors due to tracking transients. The estimation errors are also shown to be quite small in the second and third plots of Fig. 11. 

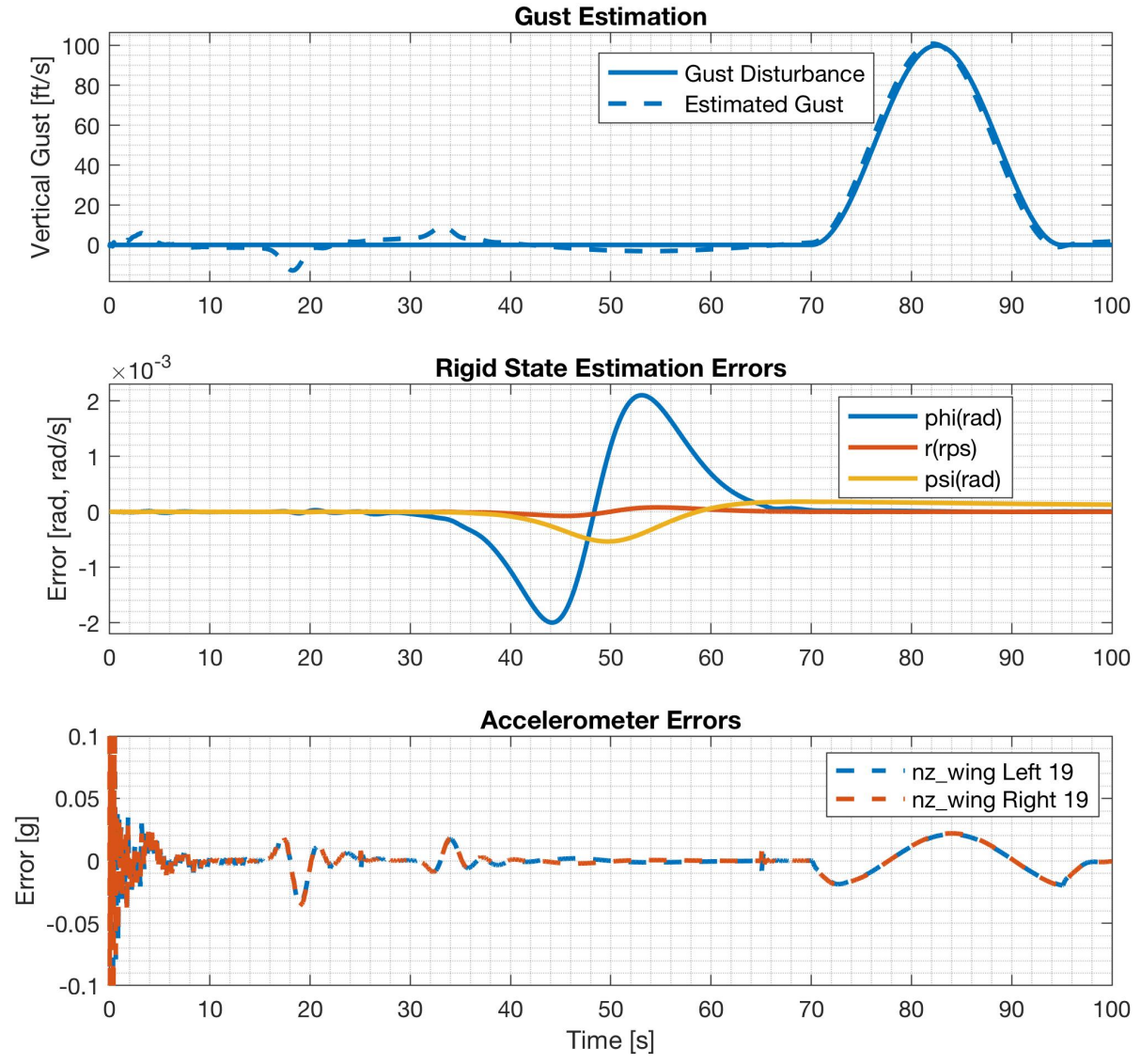

Figure 11. Extended state observer with gust estimation performance. 
The purpose of the multi-objective controller is to suppress structural modes, reduce wing root bending moment, and reduce drag. Structural mode suppression is achieved by selectively weighting portions of the $Q$ matrix in Eq. 32 corresponding to the structural mode states. Although this flight regime is below the flutter boundary, a small amount of mode suppression has been added to improve the controller's stability. Wing root bending moment and drag reduction is achieved with $q_{M}$ and $q_{D}$ weights respectively. Figure 12 shows the reduction in the square of the wing root bending moment achieved by setting $q_{M}=2 e-12$.
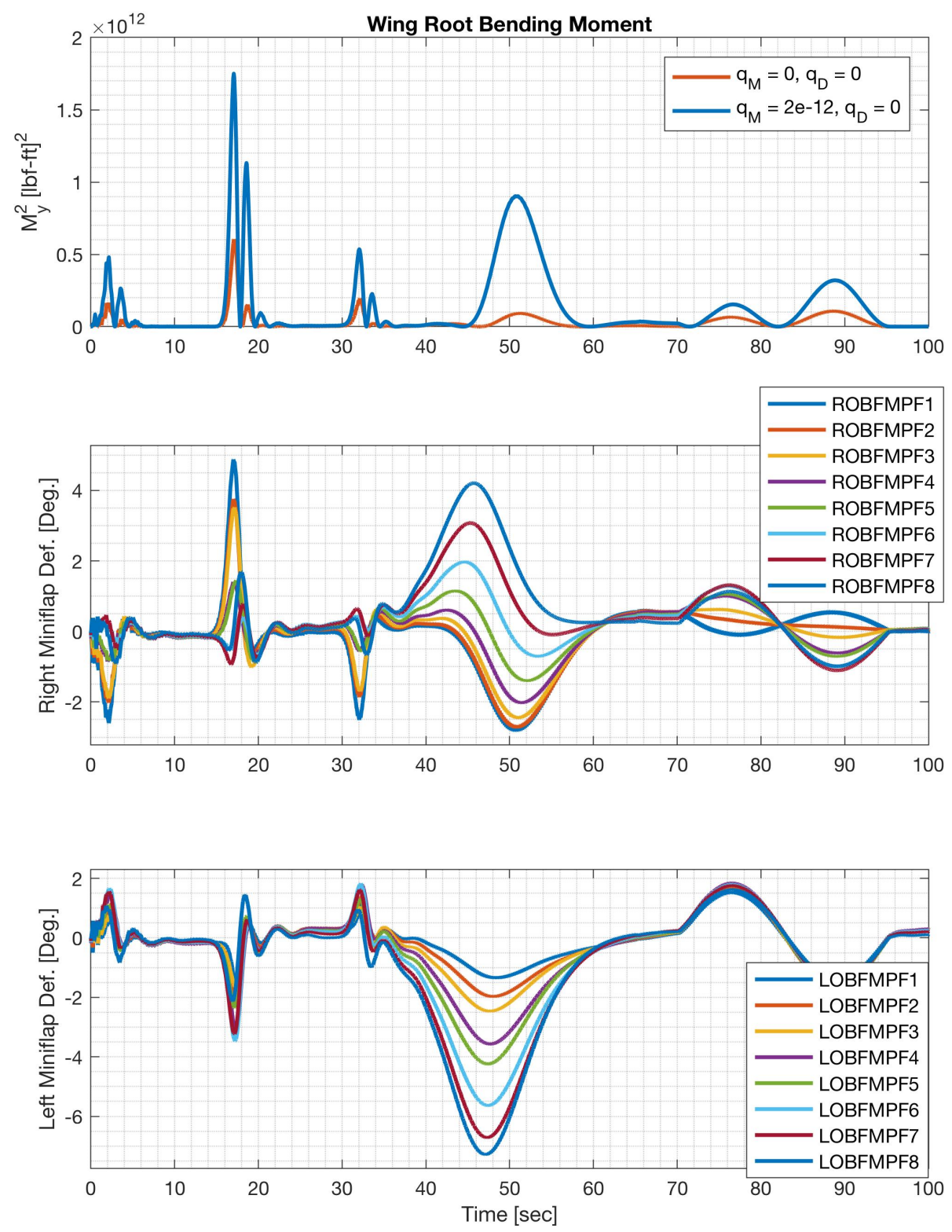

Figure 12. Wing Root Bending Moment Reduction. Miniflap deflections shown for the case when $q_{M}=2 e-12$, $q_{D}=0$.

Drag reduction tends to be a competing objective relative to wing root bending moment, so as $q_{D}$ is increased, wing root bending moment significantly increases. Also, since the drag model used here is based on a nearly optimal trim condition approximation, it is very difficult to observe much of a reduction with 
the multi-objective controller. However, minor drag reduction can be observed in Fig. 13 by zooming in on the drag coefficient plot. Note the enforcement of the miniflap deflection limits at 18 seconds.

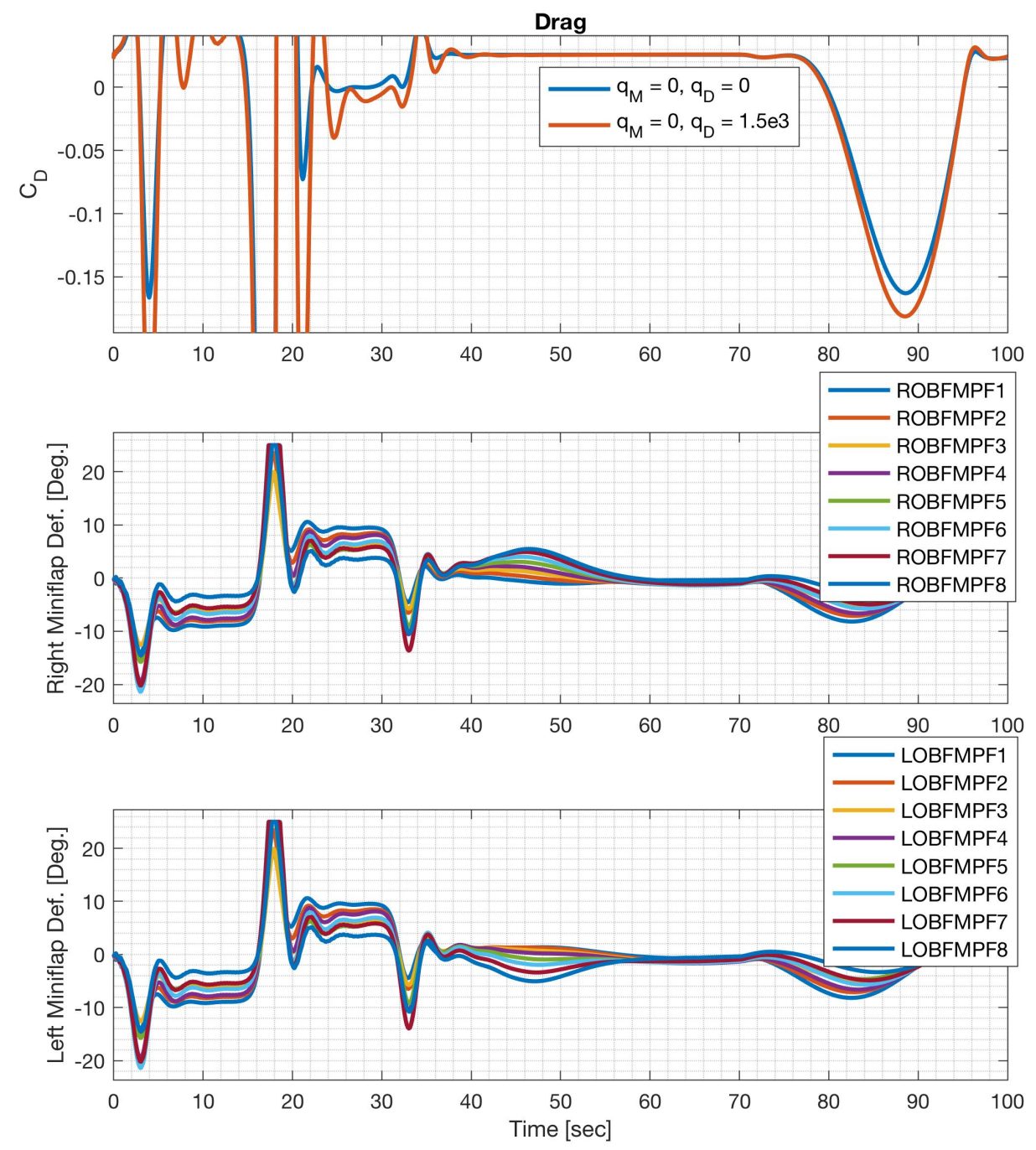

Figure 13. Drag Reduction. Miniflap deflections shown for the case when $q_{M}=0, q_{D}=1.5 e 3$. 


\section{Conclusions and Future Work}

The 6-DoF CRM-13.5 model is one of the latest models developed for the purpose of performing research in the field of aeroservoelasticity. It represents a class of aircraft with an efficient wing design, and nonconventional control surfaces tailored to provide multi-objective control opportunities to engineers. The simulation model presented in this paper will serve as a foundational platform for designing and testing additional controllers while more fidelity and analysis is carried out. This paper presented some preliminary results of 6-DoF tracking control augmented with a multi-objective controller tasked with modal suppression, as well as wing root bending moment and drag reduction. Methods of model reduction for the purpose of estimation and control were explored, and an extended state observer that generates state and gust disturbance estimation was presented and shown to work well when utilized for flutter suppression and multi-objective control.

Extensions of the work of this paper are already underway. For instance, multi-objective control with time-varying weights is presented in Ref. 8. Methods of applying gust disturbance rejection control in conjunction with the multi-objective control can also be looked at. One of the challenges of working with this particular model is the process of selecting outputs and weights that provide good state and gust estimates. This has largely been done with trial-and-error and intuition. A more analytical approach needs to be developed that will make the design and tuning phase more efficient. Also, now that the basic tracking and multi-objective controllers are in place, a more thorough stability analysis is called for. Finally, there has not been a formal trade study looking at control surface allocation for the various control objectives. This would be another valuable area of research to be conducted on this model.

\section{Acknowledgments}

The authors would like to thank Jeffrey Jonokuchi and The Boeing Research \& Technology Division for providing the CRM-13.5 models and documentation, and for their continued involvement in this work. We would also like to thank the NASA Advanced Air Transport Technology Project for support of this work.

\section{References}

\footnotetext{
${ }^{1}$ Nguyen, N., Trinh, K., Reynolds, K., Kless, J., Aftosmis, M., and Urnes, J., "Elastically shaped wing optimization and aircraft concept for improved cruise efficiency," 51st AIAA Aerospace Sciences Meeting Including the New Horizons Forum and Aerospace Exposition, 2013, p. 141.

${ }^{2}$ Nguyen, N. T., Ting, E., Chaparro, D., Drew, M. C., and Swei, S. S.-M., "Multi-Objective Flight Control for Drag Minimization and Load Alleviation of High-Aspect Ratio Flexible Wing Aircraft," 58th AIAA/ASCE/AHS/ASC Structures, Structural Dynamics, and Materials Conference, 2017, p. 1589.

${ }^{3}$ Rivers, M. B. and Dittberner, A., "Experimental Investigations of the NASA Common Research Model in the NASA Langley National Transonic Facility and NASA Ames 11-ft Transonic Wind Tunnel (Invited)," 49th AIAA Aerospace Sciences Meeting including the New Horizons Forum and Aerospace Exposition, Orlando, Florida, 4-7 January 2011.

${ }^{4}$ Vassberg, J. C. and Rivers, M. S., "Development of a Common Research Model for Applied CFD Validation Studies," 26th AIAA Applied Aerodynamics Conference, Honolulu, Hawaii, 18-21 August 2008.

${ }^{5}$ Brooks, T. R., Kenway, G. K. W., and Martins, J. R. R., "Undeflected Common Research Model (uCRM): An Aerostructural Model for the Study of high Aspect Ratio Transport Aircraft Wings," 35th AIAA Applied Aerodynamics Conference, Denver, Colorado, 5-9 June 2017.

${ }^{6}$ Hashemi, K. E., Nguyen, N. T., Drew, M., Chaparro, D., and Ting, E., "Performance Optimizing Gust Load Alleviation Control of Flexible Wing Aircraft," AIAA Guidance, Navigation, and Control Conference, Kissimmee, Florida, 8-12 January 2018.

${ }^{7}$ Nguyen, N. T., Hashemi, K. E. T., and Drew, M. C., "Multi-Objective Adaptive Control for Load Alleviation and Drag Minimization of Flexible Aircraft," AIAA Guidance, Navigation, and Control Conference, Kissimmee, Florida, 8-12 January 2018.

${ }^{8}$ Hashemi, K. E., Nguyen, N. T., and Drew, M. C., "Time-Varying Weights in Multi-Objective Optimal Control for Flexible Wing Aircraft," AIAA Guidance, Navigation, and Control Conference, San Diego, California, 7-11 January 2019.
} 